Article

\title{
Analysis and Design of a Wireless Power Transfer System with Dual Active Bridges
}

\author{
Xin Liu ${ }^{1}(\mathbb{D})$, Tianfeng Wang ${ }^{1}$, Xijun Yang ${ }^{1, *}$, Nan Jin ${ }^{2}$ and Houjun Tang ${ }^{1}$ \\ 1 Key Laboratory of Control of Power Transmission and Transformation Ministry of Education, \\ Shanghai Jiao Tong University, 800 Dongchuan RD., Shanghai 200240, China; Liu_xin@sjtu.edu.cn (X.L.); \\ wangtf1992@163.com (T.W.); hjtang@sjtu.edu.cn (H.T.) \\ 2 College of Electric and Information Engineering, Zhengzhou University of Light Industry, \\ Zhengzhou 450002, China; jinnan@zzuli.edu.cn \\ * Correspondence: yangxijun@sjtu.edu.cn; Tel.: +86-138-1606-6208
}

Academic Editor: Chunhua Liu

Received: 25 August 2017; Accepted: 9 October 2017; Published: 12 October 2017

\begin{abstract}
Nowadays, work on Wireless Power Transfer (WPT) systems with dual active bridges is attracting great attention due to their low conduction losses, power regulation, load transformation and reactance compensation. However, in these studies limitations such as overall analysis, design and realization techniques of the system were not considered properly. To address the aforementioned issues, this paper presents a detailed analysis, design and realization of a Series-Series (SS) WPT system with dual active bridges, which will improve the overall performance. Three independent Phase Angles (PAs) have been analyzed and designed in this study, one PA on the primary side and the other two PAs on the secondary side. This Multiple Degrees of Phase Control (MDPC) method can achieve additional reactance compensation, load transformation and output regulation simultaneously. To realize the proposed method in practice, key implementation techniques have been investigated in detail, including additional reactance estimation, mutual inductance estimation, phase detection and synchronization. The feasibility and effectiveness of the proposed system is verified through simulation and experimental results.
\end{abstract}

Keywords: dual active bridges; parameter estimation; Multiple Degrees of Phase Control (MDPC); synchronization; Wireless Power Transfer (WPT)

\section{Introduction}

Wireless Power Transfer (WPT) systems can utilize coupling coils to transfer energy through magnetic fields, which can avoid the physical connection of charging lines. Due to their convenience and possibility of widespread applications, the WPT technique has been the research focus of many studies [1,2]. Various wireless charging devices have been invented and manufactured for use in applications such as portable consumer electronics and electric vehicles [3-6].

Compensation capacitors are indispensable elements of a WPT system [1]. These capacitors compensate the inductive reactance of the transmitting and receiving coils, and contribute significantly to inducing a meaningful amount of current needed for sufficient magnetic field generation. In order to realize strongly coupled magnetic resonance [1,7], the ideal method is to tune the transmitter and receiver at the same resonance frequency. Although capacitor arrays can be used, they require many capacitors, switches and complex control algorithms. Furthermore, high voltage stresses on the resonant capacitors require large packaging and capacitors have large manufacturing tolerances. In addition, the capacitances and the coil inductances vary due to aging. Hence, it is a challenge to select the capacitors with ideal capacitance and rated voltage [8-10]. When non-identical resonances 
occur, the additional reactance increases apparent power and causes additional losses. Thus, extra input power is required at the same load which causes a lower efficiency.

Maximum power transfer efficiency is achieved when the load matches with the system $[7,11]$. In a Series-Series (SS) WPT system, a small loading resistance results in most losses consumed by the receiving coil, whereas a large loading resistance leads to a small reflected resistance and most of the energy is wasted in the transmitting coil. In $[9,12]$, a cascaded buck-boost converter is utilized for load transformation. An improved efficiency of $78 \%$ with a $16 \mathrm{~W}$ transferred power at a distance of $10 \mathrm{~cm}$ is achieved in [12], whereas [9] realizes an efficiency of $65 \%$ when delivering $4.5 \mathrm{~W}$ power. In [13], a two-stage switching converter is applied to modulate the load, an efficiency of $60.2 \%$ is obtained for a 20-W load at $20 \mathrm{~cm}$. In [14], the concept of phase-shift and amplitude control is introduced to modify the equivalent secondary-side load impedance for efficiency enhancement and extractable power optimization. The amplitude control is realized by an additional buck converter on the receiver side. The measured maximum efficiency is $77.3 \%$ when the coupling factor is 0.6 . In [15], a front-end boost circuit and a rear-end buck circuit are employed for voltage regulation and load transformation, respectively. The optimal efficiency is $79 \%$ when $100 \mathrm{~W}$ power is delivered at $20 \mathrm{~cm}$, with $6 \%$ power loss measured in DC-DC converters. Compared with traditional systems, these topologies help to transform the load to the optimal value and contribute to improving the efficiency. However, the control of multi-stage system is complex and additional losses in the converters are inevitable. In addition, they can increase the size of the receiver. Greater focus on finding a better solution is needed.

Adding an active rectifier on the receiver side has been proposed in [14,16]. Furthermore, researchers have found that Phase Control (PC) can be employed for conduction loss reduction and bidirectional power flow regulation [17-19]. In [16], two Phase Angles (PAs) are applied to regulate dual-side resonant currents equally to reduce losses. However, the de-tuning of the WPT system is not taken into consideration and parasitic resistances are omitted in the analysis. Researchers in $[14,20,21]$ realized that PC can change the effective voltage applied on the resonant tank and the resulting equivalent impedance seen from the AC side. In [14], the equivalent impedance is regarded as an optimization function and the mathematical expression of the equivalent impedance is not presented. In [20], a semi-bridgeless active rectifier is investigated and the output power can be regulated by secondary PC. In addition, the equivalent impedance of the semi-bridgeless active rectifier is derived. Both of [14] and [20] majorly focus on the proposal of the concept and its derivation, whereas the implementation techniques are not rigorously studied. Researchers in [21] demonstrate PC from the standpoint of load transformation and additional reactance compensation that achieves a maximum efficiency rise of $10 \%$ compared with traditional diode rectifier. An Auxiliary Measurement Coil (AMC) along with a decoupling transformer is adopted to detect the additional reactance. The decoupling transformer is used to cancel out the voltage induced by the receiver side current to the measurement circuit. Therefore, two mutual inductances, one between the receiving coil and AMC and the other of the transformer, should be identical. However, mutual inductance is strongly affected by the inductances, the position of the coils and its surroundings. It is difficult to ensure that two mutual inductances have equal values in experiments. Discrepancy of mutual inductances can bring about an estimation error in principle. In addition, the measurement of mutual inductance, which is an important parameter for optimal load calculation, is not presented. Although a real-time mutual inductance estimation method has been proposed in [22], it assumes that the WPT system is tuned completely. In [23], mutual inductance estimation is implemented by detecting primary PA. Nevertheless, when the system operates at secondary resonant frequency, a large estimation error appears. Further work on additional reactance and mutual inductance estimations is required.

Synchronization is an important technique in the control of secondary PC. Researchers in [18] proposed an AMC for synchronization. However, over $20 \%$ phase error occurs at $20 \mathrm{kHz}$ when the load or the mutual inductance changes. In [21], a current sensor is used to capture the secondary resonant current for synchronization at $30 \mathrm{kHz}$. Since the current measurement and filtering process involve a significant time delay, a phase-shift circuit should be adopted to correct the resulting phase error. 
In addition, current sensors have band limits, generally lower than $100 \mathrm{kHz}$. The synchronization may be more difficult with a higher operating frequency. Therefore, improvement of the synchronization technique is necessary.

This paper presents a detailed analysis and design of a WPT system with dual active bridges. Multiple Degrees of Phase Control (MDPC) are adopted. The system has three degrees of control freedom. Primary PA is responsible for voltage regulation, and secondary PAs for load transformation as well as additional reactance compensation. The proposed additional reactance and mutual inductance estimation methods are based on DC voltage and current samplings, which are easy to be implemented. An effective phase detection technique is elaborated where the phase information is modulated onto a DC voltage signal. In addition, a novel synchronization technique involving a comparator and an isolator is proposed that works satisfactorily at a frequency of $100 \mathrm{kHz}$. It is simpler and cheaper than previous methods and can withstand parameter variations.

\section{Modeling of Wireless Power Transfer (WPT) System}

The schematic and equivalent circuit of the proposed system are shown in Figure 1. In this paper, bold italic letters represent the phasors, whereas italic letters symbolize real numbers or Root-Mean-Square (RMS) values of the phasors. $V_{\text {in }}$ is the input voltage and $I_{\text {in }}$ is the input current. $C_{f}$ is the film capacitor which makes the inverter decoupled and the input current stable. $Q_{1}-Q_{8}$ are the Metal-Oxide-Semiconductor Field-Effect Transistors (MOSFETs) of two active bridges. $L_{1}$ and $L_{2}$ are the inductances of the transmitting and receiving coils, whose parasitic resistances are $R_{1}$ and $R_{2}$, respectively. $C_{1}$ and $C_{2}$ are the compensation capacitors, and $C_{o}$ is the output filtering capacitor. $R_{L}$ represents the loading resistance and $V_{o}$ is the voltage across it. $Z_{e}$ is the equivalent impedance, whose real part and imaginary part are denoted as $R_{e}$ and $X_{e}$, respectively. The primary resonant angular frequency $\omega_{1}$, the secondary resonant angular frequency $\omega_{2}$, the primary reactance $X_{1}$, and the secondary reactance $X_{2}$ are defined as:

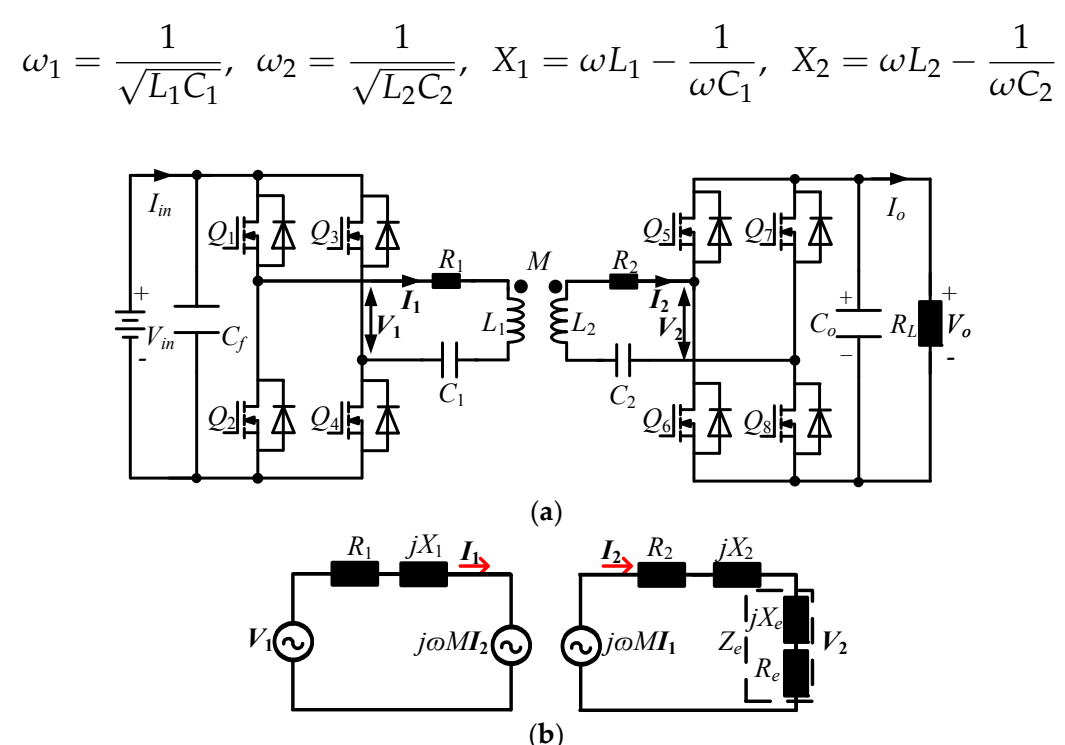

(b)

Figure 1. Proposed multiple degrees of phase control in a WPT system: (a) schematic of the proposed system; (b) equivalent circuit of the proposed system.

Uncontrolled rectification is utilized in most WPT systems $[7,9,12,24]$. The analysis presented is based on full load conditions and the output filtering capacitor is supposed to be large enough to maintain a stable output DC voltage. Thus, the equivalent impedance $Z_{e}$ is purely resistive $[7,9]$ :

$$
Z_{e}=R_{e}=\frac{8}{\pi^{2}} R_{L}
$$


The system operates at primary resonance frequency $\left(f=f_{1}\right)$, that is, $X_{1}=0$. Based on the Kirchhoff voltage law, current loops are drawn as:

$$
\left[\begin{array}{cc}
R_{1} & j \omega M \\
j \omega M & j X_{2}+R_{2}+R_{e}
\end{array}\right]\left[\begin{array}{c}
\boldsymbol{I}_{1} \\
-\boldsymbol{I}_{2}
\end{array}\right]=\left[\begin{array}{c}
\boldsymbol{V}_{1} \\
0
\end{array}\right]
$$

The power transfer efficiency $\eta$ is defined as:

$$
\eta=\frac{I_{2}^{2} R_{e}}{\operatorname{Re}\left(V_{1} I_{1}\right)}
$$

where Re represents the real part of the phasor.

By combining Equations (3) and (4), $\eta$ is derived:

$$
\eta=\frac{\omega^{2} M^{2} R_{e}}{R_{1}\left[\left(R_{2}+R_{e}\right)^{2}+X_{2}^{2}\right]+\omega^{2} M^{2}\left(R_{2}+R_{e}\right)}
$$

The three dimensional plot of the power transfer efficiency $\eta$ as a function of the load $R_{L}$ and the additional reactance $X_{2}$ is shown in Figure 2. The related parameters are listed in Table 1. The efficiency increases with the increase of $\left|X_{2}\right|$ at a constant $R_{L}$. The existence of additional reactance causes a larger apparent power, resulting in more losses consumed by the parasitic resistances. Thus, the higher $\left|X_{2}\right|$ and the lower $\eta$. By taking partial derivative of $X_{2}$, it is deduced that $\eta$ reaches the peak when $X_{2}=0$. However, the efficiency keeps varying with the load. The calculated efficiency is $49.9 \%$ when $R_{L}=998 \Omega$, which is required for maximum power transfer capability. Whereas, the efficiency is $95.2 \%$ when $R_{L}=24.8 \Omega$, which is required for maximum power transfer efficiency [9]. Figure 2 clearly illustrates the importance of additional reactance compensation and load transformation for efficiency enhancement.

Table 1. Key parameters of the proposed system.

\begin{tabular}{ccc}
\hline Symbol & Quantity & Value \\
\hline$L_{1}, L_{2}$ & inductances of transmitting and receiving coils & $475 \mu \mathrm{H}, 454 \mu \mathrm{H}$ \\
$C_{1}$ & primary compensation capacitor & $5.3 \mathrm{nF}$ \\
$C_{2}$ & secondary compensation capacitor & {$[5.3,6.0] \mathrm{nF}$} \\
$R_{1}, R_{2}$ & parasitic resistances & $0.5 \Omega, 0.5 \Omega$ \\
$f$ & operating frequency & $100 \mathrm{kHz}$ \\
$M$ & mutual inductance & $32 \mu \mathrm{H}$ \\
$R_{L}$ & loading resistance & {$[20,100] \Omega$} \\
$V_{\text {in }}$ & input voltage & $30 \mathrm{~V}$ \\
\hline
\end{tabular}

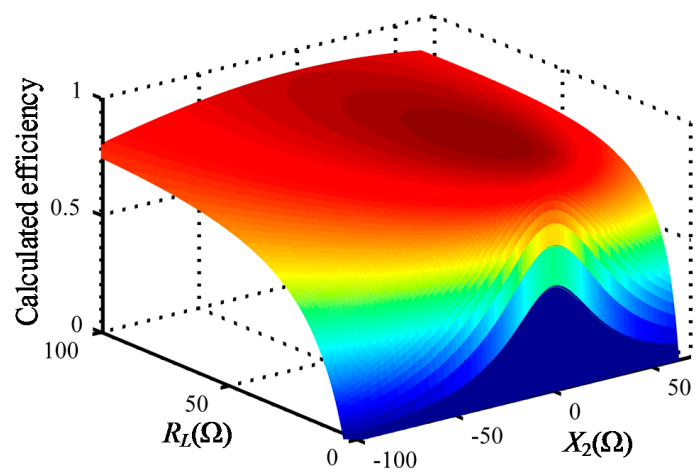

Figure 2. Three dimensional plot of the power transfer efficiency $\eta$ versus the load $R_{L}$ and the additional reactance $X_{2}$.

The optimal equivalent resistance $R_{e O P T}$ can be deduced by taking partial derivative of $R_{e}[7,9]$ : 


$$
R_{e O P T}=R_{2} \sqrt{1+\frac{\omega^{2} M^{2}}{R_{1} R_{2}}}
$$

\section{Multiple Degrees of Phase Control}

Figure 3 shows the square-wave voltages, fundamental voltages and resonant currents of two active bridges used in the SS WPT system. As can be seen from Figure 3, there are two PAs in each bridge, thus the total PAs of the system are four. $\theta$ is the PA between primary fundamental voltage and $\boldsymbol{I}_{\mathbf{1}} . \varphi$ is the PA between the secondary fundamental voltage and $\boldsymbol{I}_{\mathbf{2}} . \alpha$ and $\beta$ are the PAs of the primary and secondary bridge arms, respectively. $\varphi$ changes the impedance of the receiver and the corresponding primary reflected load, resulting in the alteration of $\theta$. Hence, there only exist three independent and controllable PAs, they are: $\alpha, \beta$ and $\varphi$. Voltage regulation, load transformation and additional reactance compensation can be realized simultaneously by full utilization of these three PAs.

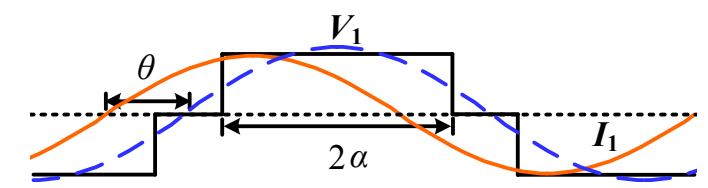

(a)

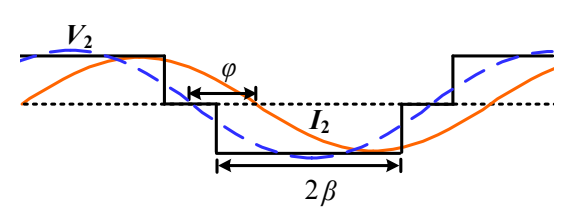

(b)

Figure 3. Square-wave voltages (black lines), fundamental voltages (blue dashed lines) and resonant currents (orange lines) in a SS WPT system: (a) primary active bridge; (b) secondary active bridge.

\subsection{Equivalent Impedance Derivation}

The equivalent impedance of the transmitter $\left(Z_{i n}\right)$ is:

$$
Z_{i n}=R_{1}+\frac{\omega^{2} M^{2}}{R_{2}+R_{e}+j\left(X_{2}+X_{e}\right)}
$$

Thus, $\theta$ can be affected by the reflected resistive and reactive components back on the primary side. In other words, there are only three independent and controllable PAs including $\alpha, \beta$ and $\varphi$. When the secondary additional reactance is compensated $\left(X_{2}+X_{e}=0\right), Z_{i n}$ is resistive even when $X_{e} \neq 0$. According to (6), the optimal load depends on the mutual inductance. When $M$ changes due to the offset of the coils, the corresponding optimal PAs vary. This paper aims to reduce the efficiency degradation caused by parameter variations. On-line estimations can be applied to detect these changes, which can adapt to the mutual inductance and load variations.

Some systems operate at $\omega_{2}$ and regulate the power flow by the PA between the primary and secondary voltage phasors $[17,25]$. It can introduce reactive power into the system and bring about undesired efficiency degradation. The proposed system operates at $\omega_{1}$ and utilizes $\varphi$ to compensate secondary additional reactance $X_{2}$. Power regulation and load transformation are realized by regulation of $\alpha$ and $\beta$, respectively. This strategy reduces the reactive power that would otherwise be present.

To obtain the optimal efficiency point, the additional reactance should be compensated completely and the load should be transformed to the optimal value. Hence, the mathematical expressions of the equivalent reactance and resistance of the receiver should be derived.

Figure 4 shows the simulated waveforms of primary resonant voltage $V_{\mathbf{1}}$ and resonant current $I_{1}$, secondary voltage $V_{2}$ and resonant current $I_{2}$, the control signals and the corresponding PAs, where $\alpha$ and $\beta$ are determined when the gate drive signals are valid simultaneously. When the upper switches $\left(Q_{1}\right.$ and $Q_{3}$ of the primary bridge, $Q_{5}$ and $Q_{7}$ of the secondary bridge) or the lower switches ( $Q_{2}$ and $Q_{4}$ of the primary bridge, $Q_{6}$ and $Q_{8}$ of the secondary bridge) are turned on, the DC voltage is disconnected from the system. Thus, the corresponding voltage $\left(V_{\mathbf{1}}\right.$ or $\left.V_{\mathbf{2}}\right)$ is zero. More details about this commutation can be found in [21]: 


$$
\mathbf{I}_{\mathbf{2}}=\frac{V_{\mathbf{1}}-\mathbf{I}_{\mathbf{1}} R_{1}}{-j \omega M} \approx \frac{V_{\mathbf{1}}}{-j \omega M}
$$

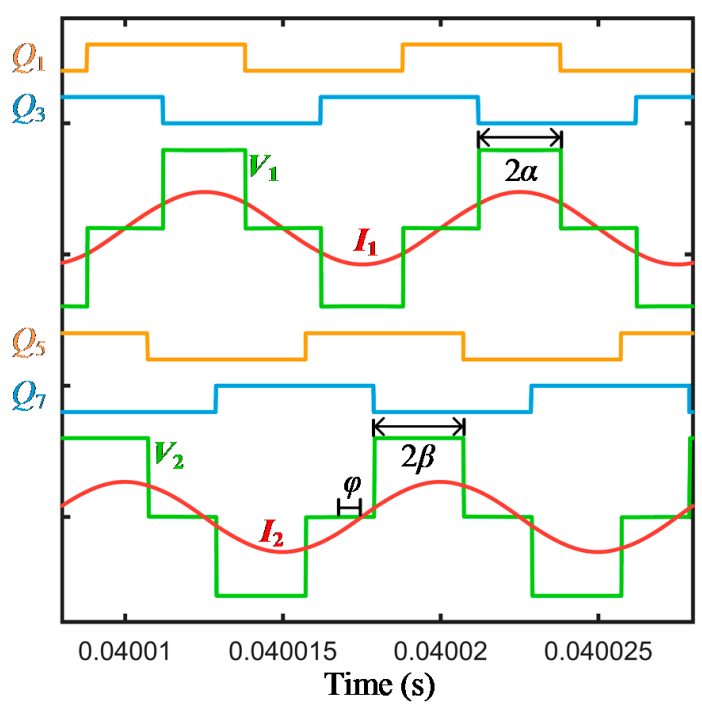

Figure 4. Simulated waveforms of the proposed MDPC.

Since the voltage drop across $R_{1}$ is small, the above equation becomes true. The SS WPT system maintains the output current, i.e., $\boldsymbol{I}_{\mathbf{2}}$ remains unchanged despite load variation or secondary PC. Therefore, the control of $V_{2}$ is equivalent to that of $\varphi$, which can be realized by shifting $Q_{5}-Q_{8}$ with respect to $\boldsymbol{I}_{\mathbf{2}}$. The left shift of $Q_{5}-Q_{8}$ brings about a positive $\varphi$, whereas a right shift brings about a negative $\varphi$. The secondary voltage $\boldsymbol{V}_{\mathbf{2}}$ can be written as (9):

$$
V_{2}=\frac{2 \sqrt{2}}{\pi} V_{o} e^{j \varphi} \sin \beta
$$

Thus, $\beta$ and $\varphi$ can change the effective voltage applied on the resonant tank and regulate the bidirectional power flow. Essentially, $\beta$ and $\varphi$ can change the equivalent impedance $Z_{e}$ in the unidirectional WPT system.

The power consumed by the load is:

$$
P_{o}=\frac{V_{o}^{2}}{R_{L}}
$$

and the input active power of the converter is:

$$
P_{2}=\left|V_{2}\right|\left|I_{2}\right| \cos \varphi
$$

Neglecting the losses of the switches, the following equation results:

$$
\left|\boldsymbol{V}_{2}\right|\left|\boldsymbol{I}_{2}\right| \cos \varphi=\frac{V_{o}^{2}}{R_{L}}
$$

Then, $I_{2}$ can be obtained by:

$$
I_{2}=\frac{\pi V_{o}}{2 \sqrt{2} R_{L} \sin \beta \cos \varphi}
$$

The equivalent impedance of the secondary active bridge can be defined as:

$$
Z_{e}=\frac{V_{2}}{I_{2}}=R_{e}+j X_{e}
$$


Thus, the equivalent resistance and reactance versus the PAs and the load are derived by substituting Equations (9) and (13) into (14):

$$
\begin{aligned}
& R_{e}=\frac{8}{\pi^{2}} R_{L} \cos ^{2} \varphi \sin ^{2} \beta \\
& X_{e}=\frac{4}{\pi^{2}} R_{L} \sin 2 \varphi \sin ^{2} \beta
\end{aligned}
$$

When $\beta=90^{\circ}$ and $\varphi=0^{\circ}$, secondary active bridge acts as a synchronous rectifier, thus Equation (15) can be simplified as (2) and $X_{e}$ is zero.

\subsection{Voltage Regulation}

Primary resonant current $\boldsymbol{I}_{\mathbf{1}}$ is derived as:

$$
\boldsymbol{I}_{\mathbf{1}}=\frac{\boldsymbol{V}_{\mathbf{1}}}{R_{1}+Z_{i n}}=\frac{\left[j\left(X_{2}+X_{e}\right)+\left(R_{e}+R_{2}\right)\right] \boldsymbol{V}_{\mathbf{1}}}{R_{1}\left[j\left(X_{2}+X_{e}\right)+\left(R_{e}+R_{2}\right)\right]+\omega^{2} M^{2}}
$$

According to voltage-divider theorem on the receiver side, the voltage across $R_{e}\left(V_{e}\right)$ is deduced:

$$
V_{e}=j \omega M I_{1} \cdot \frac{R_{e}}{j\left(X_{2}+X_{e}\right)+R_{e}+R_{2}}=\frac{j \omega M R_{e} V_{1}}{R_{1}\left[R_{2}+R_{e}+j\left(X_{2}+X_{e}\right)\right]+\omega^{2} M^{2}}
$$

The RMS value of the primary voltage $V_{\mathbf{1}}$ is:

$$
V_{1}=\frac{2 \sqrt{2}}{\pi} V_{i n} \sin \alpha
$$

Since, $V_{e}^{2} / R_{e}=V_{o}^{2} / R_{L}$, the desired $\alpha$ for voltage regulation can be obtained as:

$$
\alpha=\arcsin \frac{\pi V_{o} \sqrt{\left[\omega^{2} M^{2}+R_{1}\left(R_{e}+R_{2}\right)\right]^{2}+R_{1}^{2}\left(X_{e}+X_{2}\right)^{2}}}{2 \omega M V_{i n} \sqrt{2 R_{e} R_{L}}}
$$

When the reactance $X_{2}$ is compensated completely by $X_{e},(20)$ can be simplified as (21):

$$
\alpha=\arcsin \frac{\pi V_{o}\left[\omega^{2} M^{2}+R_{1}\left(R_{e}+R_{2}\right)\right]}{2 \omega M V_{i n} \sqrt{2 R_{e} R_{L}}}
$$

\subsection{Load Transformation and Additional Reactance Compensation}

Load transformation and additional reactance compensation can be realized only with the utilization of secondary PAs. According to Equations (15) and (16), the load transformation ratio $m$ and reactance compensation ratio $n$ are defined for normalization, respectively:

$$
\begin{aligned}
& m=\frac{R_{e}}{R_{L}}=\frac{8}{\pi^{2}} \cos ^{2} \varphi \sin ^{2} \beta \\
& n=\frac{X_{e}}{R_{L}}=\frac{4}{\pi^{2}} \sin 2 \varphi \sin ^{2} \beta
\end{aligned}
$$

where $m$ ranges from 0 to $\frac{8}{\pi^{2}}$. It is single-peaked and the peak value is achieved when $\beta=90^{\circ}$ and $\varphi=0^{\circ}$, as shown in Figure 5a. $n$ has one peak and one valley that ranges from $-\frac{4}{\pi^{2}}$ to $\frac{4}{\pi^{2}}$, as shown in Figure $5 b$.

With the proposed rectifier, the efficiency $\eta$ is derived as:

$$
\eta=\frac{\omega^{2} M^{2} R_{e}}{R_{1}\left[\left(R_{2}+R_{e}\right)^{2}+\left(X_{2}+X_{e}\right)^{2}\right]+\omega^{2} M^{2}\left(R_{2}+R_{e}\right)}
$$


As can be seen from (24), the existence of $\left(X_{2}+X_{e}\right)^{2}$ decreases the efficiency. Regarding $\left(X_{2}+X_{e}\right)$ as a variable and taking partial derivative of it, it can be deduced that $\eta$ reaches the peak when $X_{2}+X_{e}=0$. By taking partial derivative of $R_{e}$, the same expression of $R_{e O P T}$ can be obtained.

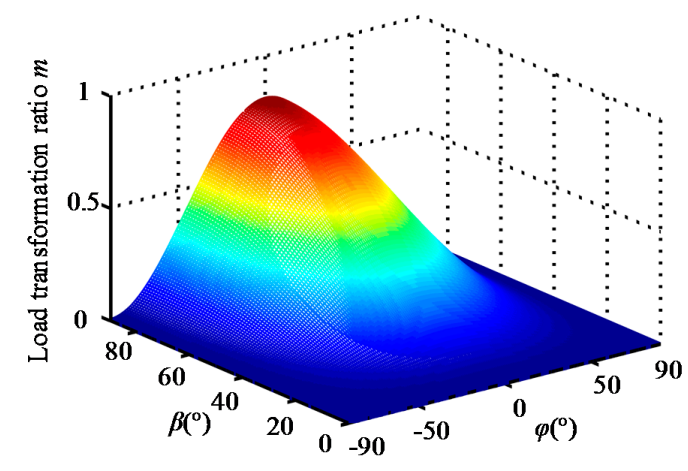

(a)

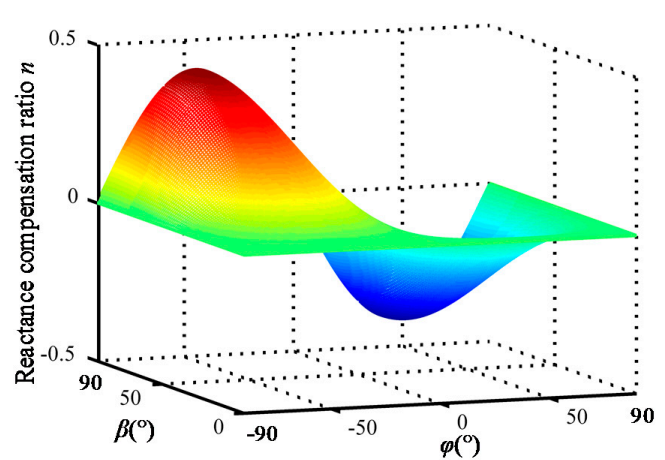

(b)

Figure 5. Load transformation ratio $m$ and reactance compensation ratio $n$ versus $\varphi$ and $\beta$ : (a) load transformation ratio; (b) reactance compensation ratio.

The optimal equivalent resistances of the proposed system and the uncontrolled rectification system are the same. To meet the requirements of load transformation and reactance compensation, Equations (25) and (26) should be satisfied:

$$
\begin{gathered}
R_{e}=R_{e O P T} \\
X_{2}+X_{e}=0
\end{gathered}
$$

Substituting (15) and (16) into (25) and (26), the following equations are obtained:

$$
\begin{aligned}
& \frac{8}{\pi^{2}} R_{L} \cos ^{2} \varphi \sin ^{2} \beta=R_{e O P T} \\
& X_{2}+\frac{4}{\pi^{2}} R_{L} \sin 2 \varphi \sin ^{2} \beta=0
\end{aligned}
$$

When the load and additional reactance fall into the available regulation range, (27) and (28) can be solved. The values of $\varphi$ and $\beta$ are:

$$
\begin{gathered}
\varphi=\operatorname{acrtan}\left(\frac{-X_{2}}{R_{e O P T}}\right) \\
\beta=\arcsin \sqrt{\frac{\pi^{2} R_{e O P T}}{8 R_{L} \cos ^{2} \varphi}}
\end{gathered}
$$

Figure 6 shows the optimal $\varphi$ and $\beta$ versus $X_{2}$ at different loads. Point A represents the case without additional reactance on the receiver side, hence $\varphi$ is zero. When $\cos \varphi=0, \beta$ is a function of $R_{L}$. Point $B$ and $C$ are the optimal angles of $\beta$ for $100 \Omega$ and $50 \Omega$, respectively. A higher load corresponds to a smaller $\beta$, whereas $\varphi$ is a function of $X_{2}$ and $R_{e_{-} \text {opt }}$, which is independent of $R_{L}$. When the load varies, the optimal $\varphi$ should remain unchanged, that is, two solid plots of $\varphi$ in Figure 6 are the same. Once the additional reactance $X_{2}$ is compensated completely by $X_{e}$, the reflected impedance of the receiver is purely resistive. Thus, $\boldsymbol{V}_{\mathbf{1}}$ and $\boldsymbol{I}_{\mathbf{1}}$ should be in phase, as shown in Figure 4. 


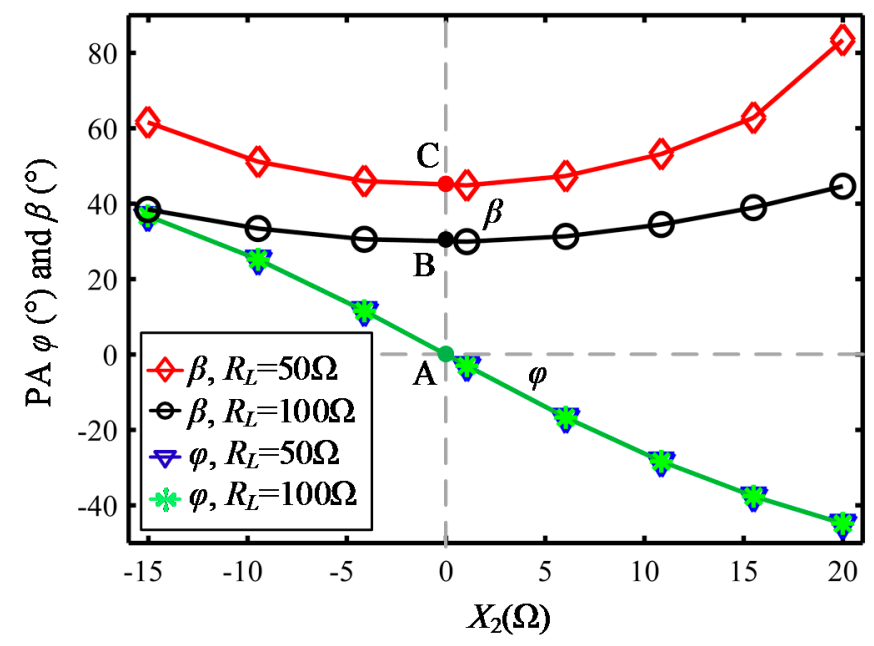

Figure 6. Optimal phase angles $\varphi$ and $\beta$ versus the additional reactance $X_{2}$.

\subsection{Additional Reactance and Mutual Inductance Estimations}

Mutual inductance and additional reactance determine the optimal values of $\varphi$ and $\beta$. Therefore, it is important to measure these parameters. In this part, easy implementation methods of additional reactance and mutual inductance estimations are elaborated.

It is assumed that primary voltage $V_{\mathbf{1}}$ lags primary current $\boldsymbol{I}_{\mathbf{1}}$ by $\theta$, as shown in Figure 3a. Then, (31) is obtained:

$$
\frac{V_{1}}{I_{1}} e^{-j \theta}=R_{1}+\frac{\omega^{2} M^{2}}{j\left(X_{e}+X_{2}\right)+R_{e}+R_{2}}
$$

The equation can be decomposed into two parts, they are:

$$
\begin{gathered}
\frac{V_{1}}{I_{1}} \cos \theta=R_{1}+\frac{\omega^{2} M^{2}\left(R_{e}+R_{2}\right)}{\left(X_{e}+X_{2}\right)^{2}+\left(R_{e}+R_{2}\right)^{2}} \\
\frac{V_{1}}{I_{1}} \sin \theta=\frac{\omega^{2} M^{2}\left(X_{e}+X_{2}\right)}{\left(X_{e}+X_{2}\right)^{2}+\left(R_{e}+R_{2}\right)^{2}}
\end{gathered}
$$

If $V_{1}$ and $I_{1}$ are in phase, i.e., $\theta=0$, the following equations are derived:

$$
\begin{gathered}
X_{e}+X_{2}=0 \\
M=\sqrt{\frac{\left(V_{1}-I_{1} R_{1}\right)\left(R_{e}+R_{2}\right)}{\omega^{2} I_{1}}}
\end{gathered}
$$

Since $X_{e}$ is controlled, the value of $X_{2}$ can be obtained by:

$$
X_{2}=-X_{e}
$$

The above equations can be used to estimate $X_{2}$ in an open loop test. To adjust $\varphi$ meanwhile observe $\theta$ by oscilloscope. When $\theta=0$, it is assumed that $X_{2}$ is compensated completely and its value can be obtained according to (36).

Regardless of the value of $\theta$, the following equations are deduced for on-line estimations:

$$
X_{2}=\frac{V_{1}\left(R_{e}+R_{2}\right) \sin \theta}{V_{1} \cos \theta-I_{1} R_{1}}-X_{e} \approx\left(R_{e}+R_{2}\right) \tan \theta-X_{e}
$$




$$
M=\sqrt{\frac{\left(V_{1} \cos \theta-I_{1} R_{1}\right)\left[\left(X_{e}+X_{2}\right)^{2}+\left(R_{e}+R_{2}\right)^{2}\right]}{\omega^{2} I_{1}\left(R_{e}+R_{2}\right)}}
$$

According to (19), the value of $V_{1}$ can be obtained by sampling DC voltage $V_{i n}$. Thus, the main task of additional reactance estimation is to detect $\theta$. Since it is difficult to detect high-frequency PA directly, an indirect and simple method will be presented in Section 4.

By applying the law of conservation of energy, the input power and output power of primary inverter should be equal, that is:

$$
V_{\text {in }} I_{\text {in }}=V_{1} I_{1} \cos \theta
$$

The following equation can be obtained:

$$
I_{1}=\frac{V_{\text {in }} I_{\text {in }}}{V_{1} \cos \theta}=\frac{\pi I_{\text {in }}}{2 \sqrt{2} \sin \alpha \cos \theta}
$$

Taking (37) and (40) into (38) and neglecting $I_{1} R_{1}$, the simplified estimated $M$ can be derived:

$$
M \approx \sqrt{\frac{8 V_{i n}\left(R_{e}+R_{2}\right) \sin ^{2} \alpha}{\pi^{2} \omega^{2} I_{\text {in }}}}
$$

\section{Implementation Techniques}

The overall system block diagram is shown in Figure 7. Two Digital Signal Processor (DSP) chips (TMS320F28335) are used as the controllers. The phase detection circuit and the synchronization circuit are shown to be within the regions enclosed with yellow and cyan dotted lines, respectively. The estimations of $X_{2}$ and $M$ are implemented on the receiver side. More information of the implementation techniques will be elaborated in the following three parts.

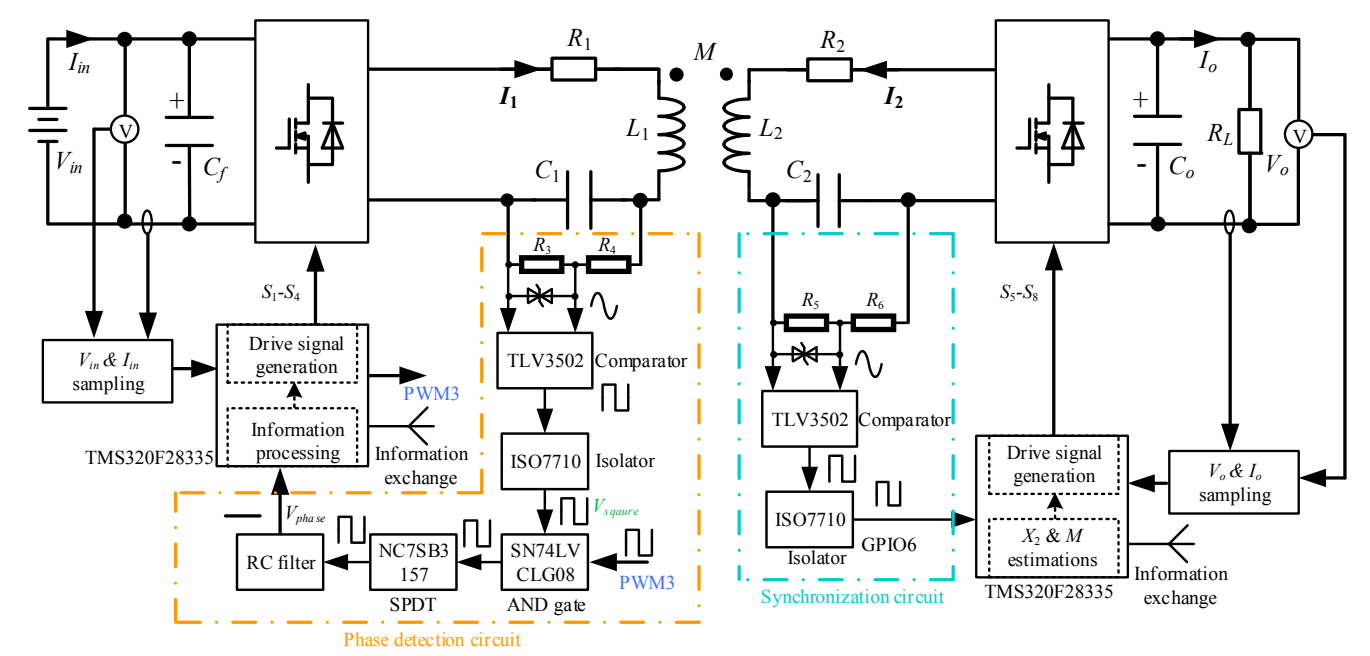

Figure 7. Overall system block diagram.

\subsection{Phase Detection}

Since direct detection of high frequency PA is difficult, the phase information is modulated onto a DC voltage signal. The phase detection circuit is shown in Figure 7 and a graphical illustration in Figure 8a. CMPA and CMPB are the values of the reserved registers, and TBPRD is the time base period. The voltage across $C_{1}\left(V_{C_{1}}\right)$ is divided by the resistors. Afterwards, the sinusoidal signal is sent to the comparator TLV3502-Q1 whose maximum operating frequency is $80 \mathrm{MHz}$. Then, a square wave is generated that goes through an isolator. PWM3 is controlled by the primary DSP. It is in phase with the fundamental voltage of $\boldsymbol{V}_{\mathbf{1}}$, as shown in Figure $8 \mathrm{a}$. The square wave $V_{\text {sqaure }}$ and PWM3 pass 
through an AND gate, which can obtain a phase detection signal. Since $\boldsymbol{I}_{\mathbf{1}}$ always leads $V_{C_{1}}$ by $90^{\circ}$, the pulse width of the phase detection signal $d$ is:

$$
d=\frac{1}{4}+\frac{1}{4} \cdot \frac{\theta}{90}
$$

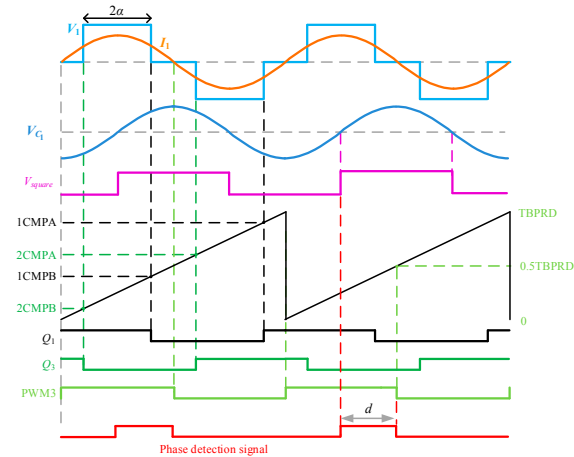

(a)

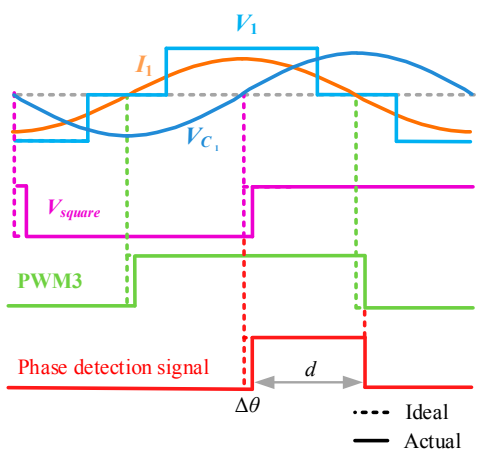

(b)

Figure 8. Generation of phase detection signal: (a) graphical illustration; (b) time delay elimination.

The signal is sent to a single-pole/double-throw (SPDT) analog switch NC7SB3157 whose operating frequency can be as high as $250 \mathrm{MHz}$. Processed by a RC filter, the phase information is modulated onto the DC voltage $V_{\text {phase }}$. The voltage can be denoted as:

$$
V_{\text {phase }}=d V_{c c}
$$

where $V_{c c}$ is the power supply of the switch.

Then, $\theta$ can be calculated by:

$$
\theta=\frac{360 V_{\text {phase }}}{V_{c c}}-90
$$

In the unidirectional WPT system, $\theta$ ranges from $-90^{\circ}$ to $90^{\circ}$. Therefore, $d$ ranges from 0 to 0.5 . The voltage corresponding to $1^{\circ}$ is $\frac{0.5 V_{c c}}{180}$. Since the valid sampling voltage of the selected DSP ranges from 0 to $3 \mathrm{~V}$, the minimum voltage that can be distinguished is $\frac{3}{4095}$. Thus, the resolution of PA is:

$$
1 L S B=\frac{3}{4095} \cdot \frac{180}{0.5 V_{c c}}=\frac{0.26}{V_{c c}}
$$

Therefore, the theoretical accuracy can be $0.078^{\circ}$ when $V_{c c}$ is $3.3 \mathrm{~V}$, which is acceptable for phase detection.

The main estimation error comes from two sources: the non-linearity of the analog chips and the time delay of the digital signal. The comparator turns the analog signal into a digital signal, whereas the analog switch turns it back. In order to improve the accuracy, high speed and high performance comparator and analog switch are chosen whose bandwidths are 800 and 2500 times greater than the operating frequency respectively, which will significantly reduce the influence of the non-linearity of the chips. The digital signal is insensitive to the noise, however, the time delay caused by the chips should be corrected as shown in Figure 8b. If PWM3 is generated without correction (as shown by the dashed line), an estimation error $\Delta \theta$ appears. This problem can be easily addressed by right shift of PWM3 via changing the corresponding register values. Since, the time delay is a constant value, a correction factor can be implemented. When $V_{\mathbf{1}}$ and $\boldsymbol{I}_{\mathbf{1}}$ are in phase, after the regulation of PWM3, $d$ should be 0.25 , i.e., $V_{\text {phase }}$ should be $0.25 V_{c c}$.

The phase detection system shown in Figure 7 has several advantages likewise, the system can be easily corrected by PWM3 which can eliminate the influence of time delay, meanwhile the 
theoretical accuracy of the system is high. Secondly, $V_{C_{1}}$ is at high level and increases with the increase of transferred power, making it resilient to interference by switching noise. These characteristics contribute to an accurate detection of the point of zero-crossing. The robustness of the system should increase with the increase of the power level. Finally, it is based on the synchronization circuit that can be easily realized especially in the bidirectional WPT systems.

\subsection{Synchronization}

Although the transmitter and the receiver are isolated, they have rigorous time sequence. Therefore, synchronization is crucial. Different from previous works that use an additional measurement coil or a current sensor, this paper presents a novel method of using the voltage across $C_{2}\left(V_{C_{2}}\right)$ for the synchronization, as shown in Figure 7. Since $V_{C_{2}}$ is at a higher level, it is not vulnerable to interference, ensuring the reliability of the synchronization circuit. At first, $V_{C_{2}}$ is divided across the resistors and a bidirectional Zener diode is paralleled to prevent overvoltage. Then, the divided sinusoidal voltage is sent to comparator TLV3502 and it can generate a square wave, indicating the zero-crossing point of $V_{C_{2}}$. After passing through isolator ISO7710, this signal is sent to EPWM synchronization port (GPIO6 in TMS320F28335). The synchronization signal is only relative to the zero-crossing point of $V_{C_{2}}$, hence this method can withstand large parameter variations including the operating frequency, the mutual inductance and the load. Since neither AMC nor the current sensor is used, this synchronization method is simpler and cheaper.

\subsection{Additional Reactance and Mutual Inductance Estimations}

Additional reactance and mutual inductance can be estimated on-line according to the algorithm shown in Figure 9. The PAs are controlled, hence they are known in advance, as well as $R_{2}$. Since $V_{\text {in }}, I_{i n}, V_{o}, I_{o}$ and $V_{\text {phase }}$ are DC signals, they can be sampled easily. $R_{L}$ is calculated by $V_{o} / I_{o}$, and $\theta$ can be obtained according to (44). Then, $R_{e}$ and $X_{e}$ can be calculated based on (15) and (16). Finally, additional reactance and mutual inductance estimations can be achieved according to Equations (37) and (41), respectively.

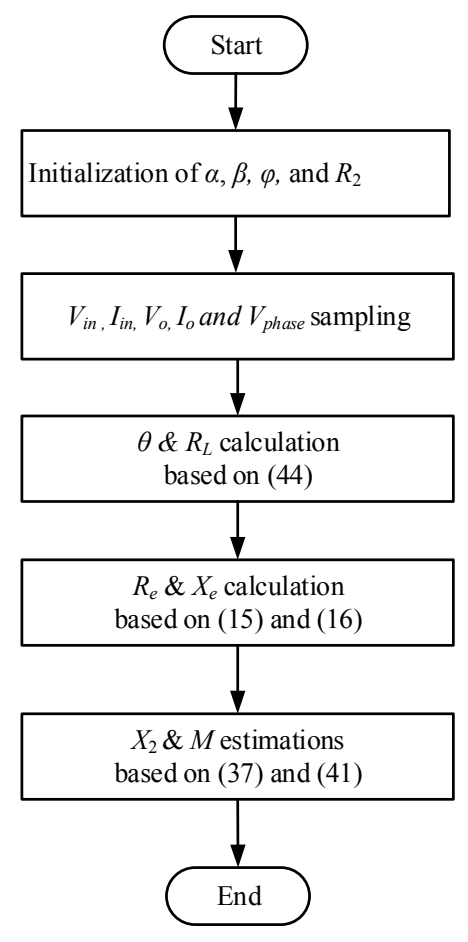

Figure 9. Flowchart of parameter estimation program. 


\section{Simulation and Experimental Results}

To verify the feasibility and effectiveness of the proposed system, a MATLAB/Simulink model and an experimental prototype are implemented. The system specifications are shown in Table 1. In addition, uncontrolled and synchronous rectifiers are added to compare with the proposed method. The synchronous rectification is a special case of $\mathrm{PC}$, in which $\beta=90^{\circ}$ and $\varphi=0^{\circ}$.

\subsection{Simulation Results}

Figure 10 shows the simulated waveforms, where uncontrolled rectifier waveforms are shown in Figure 10a, synchronous rectifier waveforms in Figure 10b and MDPC waveforms in Figure 10c. $C_{2}$ is $5.9 \mathrm{nF}$ and $R_{L}$ is $50 \Omega$. To avoid the zero current crossing issue, the switches remain off and $I_{2}$ flows through the diodes near zero current point. Thus, forward voltage of the diodes is added to $V_{2}$. The output voltage stabilizes at the desired $30 \mathrm{~V}$ by regulating $\alpha$. Although $\boldsymbol{V}_{\mathbf{2}}$ and $\boldsymbol{I}_{\mathbf{2}}$ are in phase both in Figure $10 \mathrm{~b}, \boldsymbol{I}_{\mathbf{1}}$ leads $\boldsymbol{V}_{\mathbf{1}}$. The equivalent impedance of uncontrolled and synchronous rectifiers are both resistive. However, additional inductive reactance exists on the secondary side, resulting in a reflected capacitive reactance on the primary side. Figure 10c shows the additional inductive reactance compensation results by MDPC. $\beta$ and $\varphi$ are set at $63^{\circ}$ and $-38^{\circ}$ according to the calculations of Equations (29) and (30), whereas $\alpha$ is $47^{\circ}$. Since $\boldsymbol{I}_{\mathbf{2}}$ leads $\boldsymbol{V}_{\mathbf{2}}$, the equivalent impedance has capacitive and resistive components. The load is transformed to the optimal $20.1 \Omega$ and the additional inductive reactance is compensated completely. Hence $\boldsymbol{V}_{\mathbf{1}}$ is in phase with $\boldsymbol{I}_{\mathbf{1}}$. Additional capacitive reactance can also be compensated as shown in Figure 4.

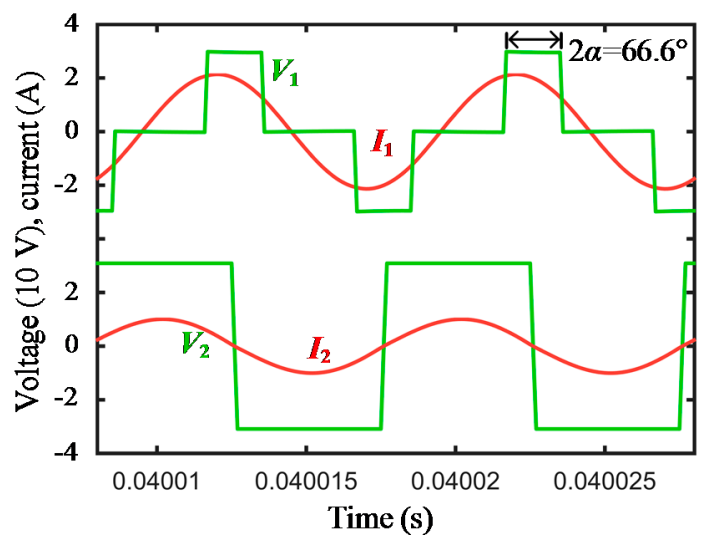

(a)

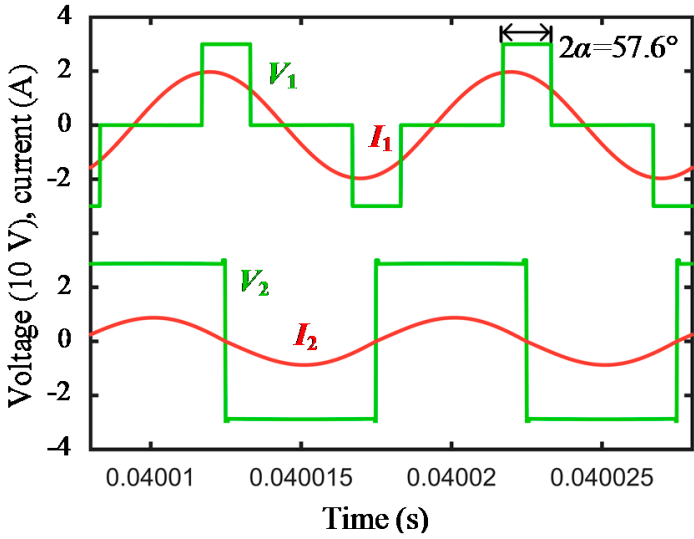

(b)

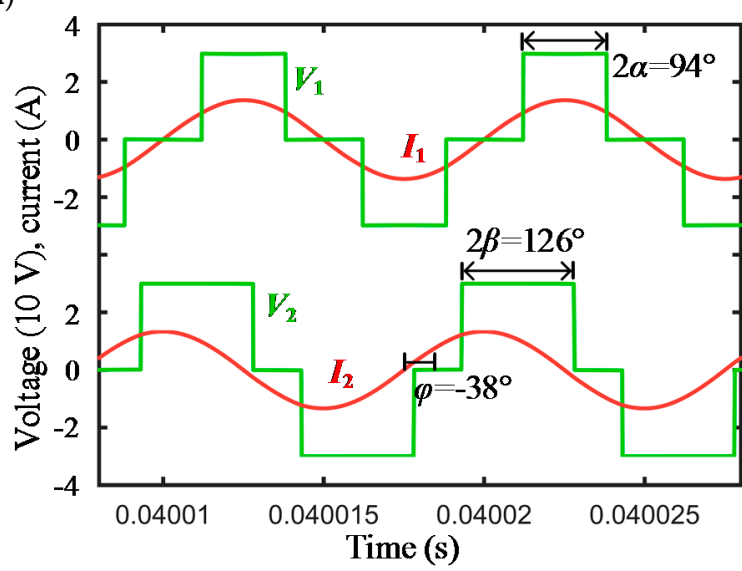

(c)

Figure 10. Simulated waveforms: (a) uncontrolled rectifier; (b) synchronous rectifier; (c) additional inductive reactance compensation and load transformation by proposed MDPC. 
Although the above results prove that $\alpha$ can be used for voltage regulation, $\alpha$ is controlled to maintain a constant output power $(18 \mathrm{~W})$ for a more accurate efficiency comparison in the simulations and experiments.

$C_{2}$ changes from $5.3 \mathrm{nF}$ to $6.0 \mathrm{nF}$. The corresponding $X_{2}$ ranges from $-15 \Omega$ to $20 \Omega$. Simulation results of $\alpha, \beta$ and $\varphi$ versus $X_{2}$ are shown in Figure 11. Although $X_{2}$ varies, the transmitter sees it as the same due to compensation through MDPC. Thus, $\alpha$ almost remains unchanged. $\beta$ and $\varphi$ change with $X_{2}$. The observed trends agree with the calculated results in Figure 6.

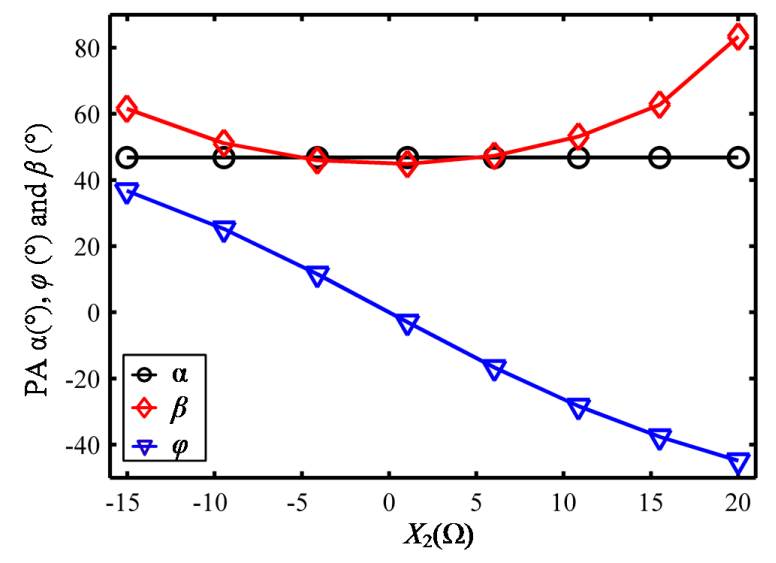

Figure 11. Simulation phase angles vs. additional reactance under MDPC.

Figure 12a shows the simulation DC-DC efficiencies of load transformation where $C_{2}$ is $5.6 \mathrm{nF}$. The equivalent impedances of the uncontrolled and synchronous rectifiers are resistive with a constant transformation ratio. Hence, uncontrolled and synchronous rectifiers just function as AC-DC converters. Although $I_{2}$ decreases with the increase of the load, resulting in lower losses on the receiver side, $\boldsymbol{I}_{\mathbf{1}}$ increases. Total losses caused by parasitic resistances increase when the load deviates from the optimal value. When the load varies in a large range, both the efficiencies of the uncontrolled and synchronous rectifier systems decrease significantly. However, the proposed MDPC method contributes to keeping the efficiency optimized. Since the load is transformed to the designed value, the influence of load mismatch is minimized. The uncontrolled rectifier system experiences the greatest efficiency drop due to the additional influence of the voltage loss in the diodes (1.6 V). For instance, if the transferred power is $18 \mathrm{~W}$ and the equivalent current flowing through two diodes is $0.67 \mathrm{~A}$, the calculated efficiency drop will be $6 \%$. Therefore, a large part of the transferred energy is consumed by the diodes.

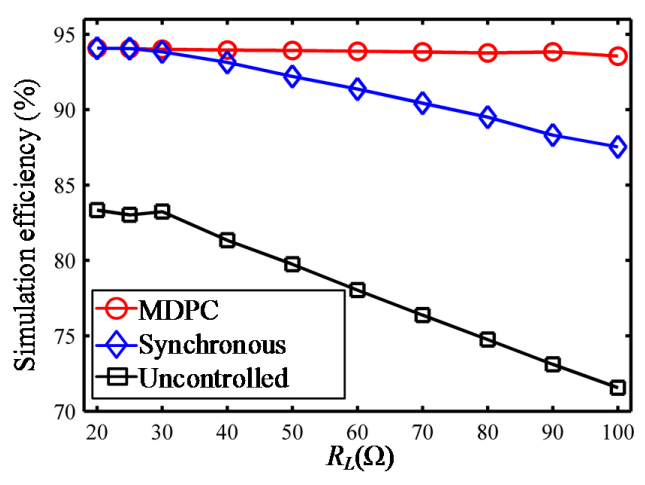

(a)

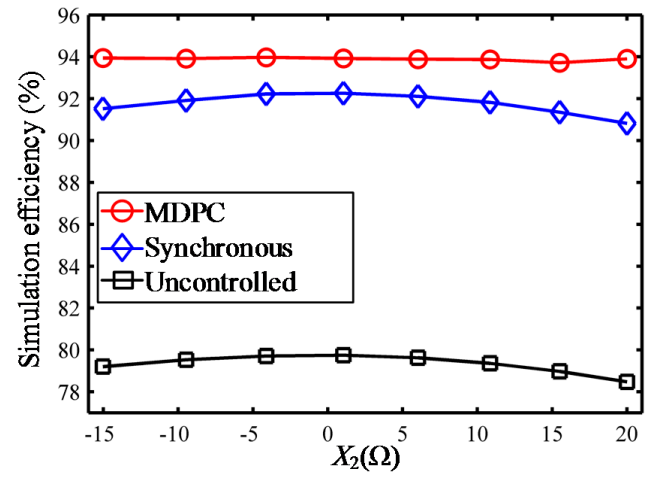

(b)

Figure 12. Simulation efficiency comparison of different methods: (a) simulation efficiency vs. load; (b) simulation efficiency vs. additional reactance. 
Figure $12 \mathrm{~b}$ shows the simulation DC-DC efficiencies of additional reactance compensation, where $R_{L}$ remains $50 \Omega$. The proposed system shows the lowest simulation efficiency of $93.9 \%$ during a large reactance variation. However, the lowest efficiencies of $87.5 \%$ and $71.6 \%$ are observed with the synchronous and uncontrolled rectifiers, respectively. Additional reactance is compensated by MDPC, hence the influence is eliminated. The existence of $X_{2}$ requires higher input power, which degrades the efficiency as indicated by Equation (5). Thus, a larger $\left|X_{2}\right|$ results in a smaller overall efficiency in the uncontrolled and synchronous rectifier systems.

\subsection{Experimental Results}

The photograph of the implemented prototype is shown in Figure 13. Primary active bridge inverts the DC voltage, thereafter, the power is transmitted through the coupling coils. Secondary active bridge converts the high frequency resonant current and the loading resistance consumes the energy. Synchronization circuit provides signal for the converter. Controllers on the primary and secondary sides generate a total of eight gate drive signals.

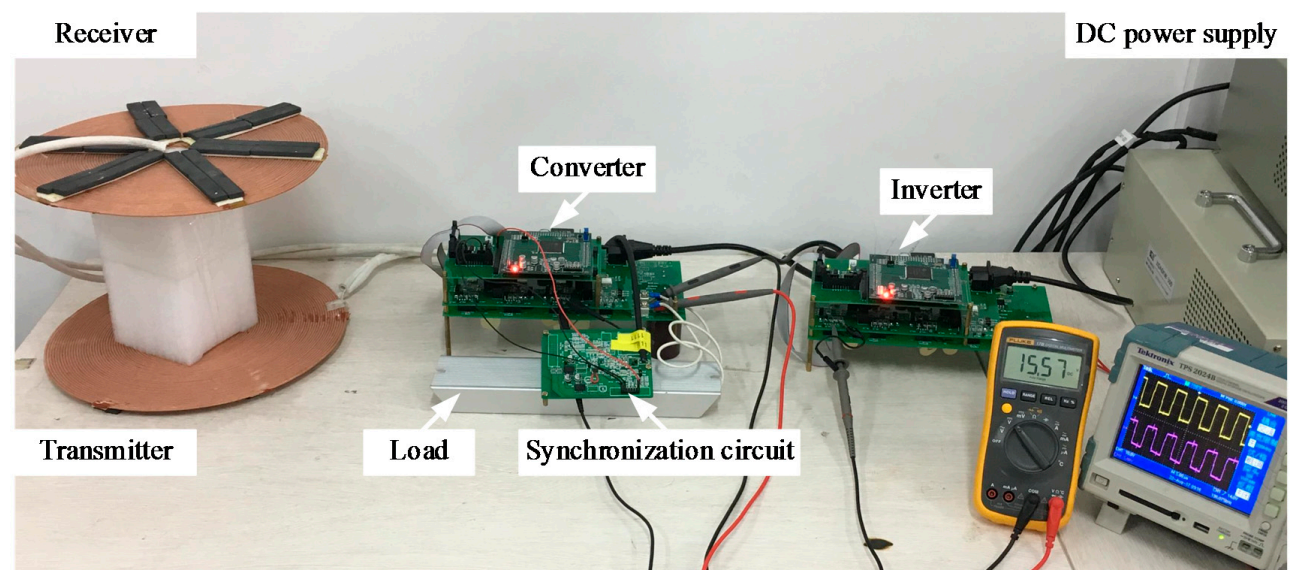

Figure 13. Photograph of the prototype.

During initialization, a program for primary resonant frequency tracking is executed, with $V_{\text {in }}$ being $2 \mathrm{~V}$. The load is removed under this step and $\theta$ is detected. The operating frequency should increase for a positive $\theta$, whereas decrease for a negative $\theta . f_{1}$ is locked when $\theta=0$, as shown in Figure 14 a. $V_{\text {phase }}$ should be $0.825 \mathrm{~V}$ theoretically.

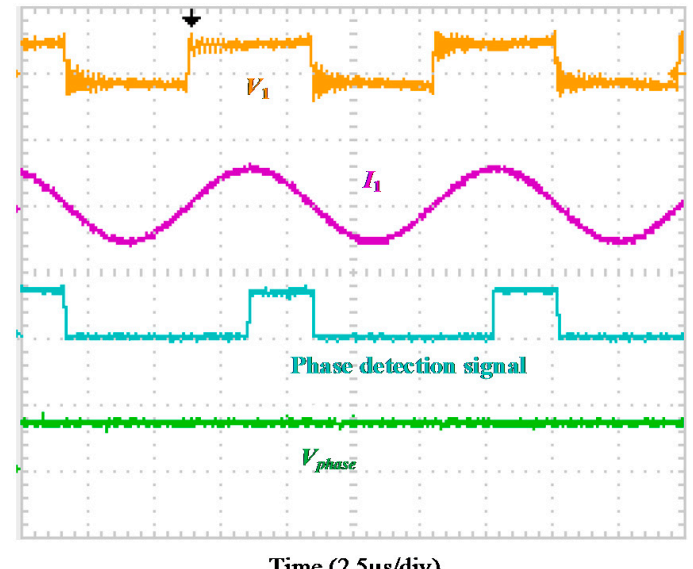

(a)

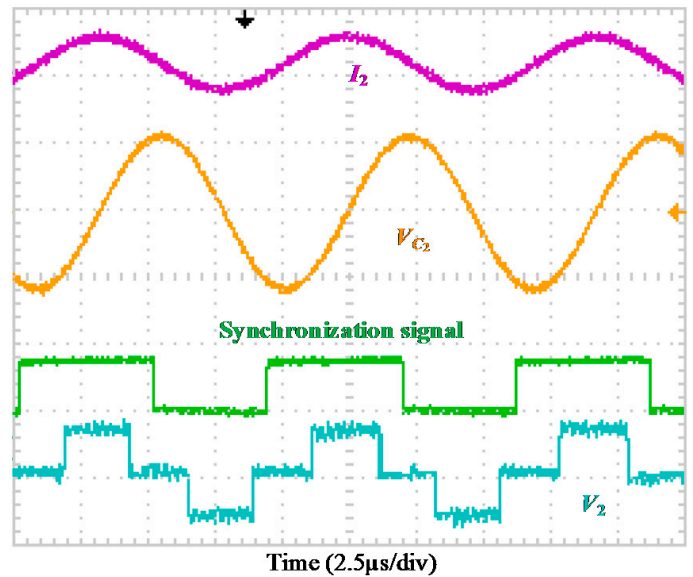

(b)

Figure 14. Cont. 


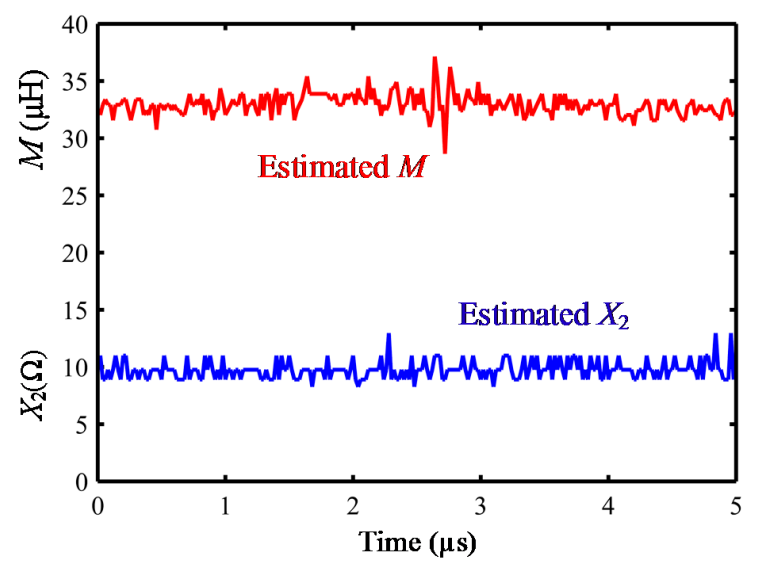

(c)

Figure 14. Experimental results: (a) phase detection; (b) synchronization; (c) additional reactance and mutual inductance estimations.

Figure $14 \mathrm{~b}$ shows the synchronization process. $\boldsymbol{I}_{2}$ flows through $C_{2}$ and produces $V_{C_{2}}$. The voltage is divided by the resistances and fed to a comparator, generating the synchronization signal. Afterwards, the secondary controller generates four gate drive signals based on it and then $V_{2}$ is obtained. Figure $14 \mathrm{c}$ shows the estimated results of $X_{2}$ and $M$. The estimated $M$ is about $32 \mu \mathrm{H}$ and $X_{2}$ is about $10 \Omega$. Figure 15 shows the experimental waveforms when $R_{L}=50 \Omega$. $C_{2}$ is $5.9 \mathrm{nF}$ in the first three experiments and $5.4 \mathrm{nF}$ in the fourth experiment. Uncontrolled and synchronous rectifier waveforms are shown in Figure 15a,b, respectively. Additional inductive reactance exists on the receiver side, thus $I_{1}$ leads $V_{1}$. Only voltage regulation is obtained by the two methods. When uncontrolled rectification is applied, $\boldsymbol{I}_{\mathbf{2}}$ and $\boldsymbol{V}_{\mathbf{2}}$ are seen to be in phase in the simulation, whereas $\boldsymbol{I}_{\mathbf{2}}$ leads $\boldsymbol{V}_{\mathbf{2}}$ in the experimental results. $\boldsymbol{V}_{\mathbf{2}}$ changes its state after $\boldsymbol{I}_{\mathbf{2}}$ crosses zero point. The discharging and charging of the parasitic capacitors needs some time, resulting in a delay in $V_{2}$. Although this phenomena can be reduced by using high performance diodes, an active rectifier used on the receiver side can address this problem completely. Additional inductive reactance compensation results is shown in Figure 15c. The additional inductive reactance is compensated, meanwhile load transformation and voltage regulation are achieved. It can be observed that $\boldsymbol{I}_{\mathbf{1}}$ in Figure $15 \mathrm{c}$ is smaller than those in Figure 15a,b. With the same input voltage, lower input current implies lower input power and therefore, lower losses. Figure $15 \mathrm{~d}$ shows the experimental results of additional capacitive reactance compensation. Although additional capacitive reactance exists, $\boldsymbol{I}_{\mathbf{1}}$ is still in phase with $V_{\mathbf{1}}$ as expected.

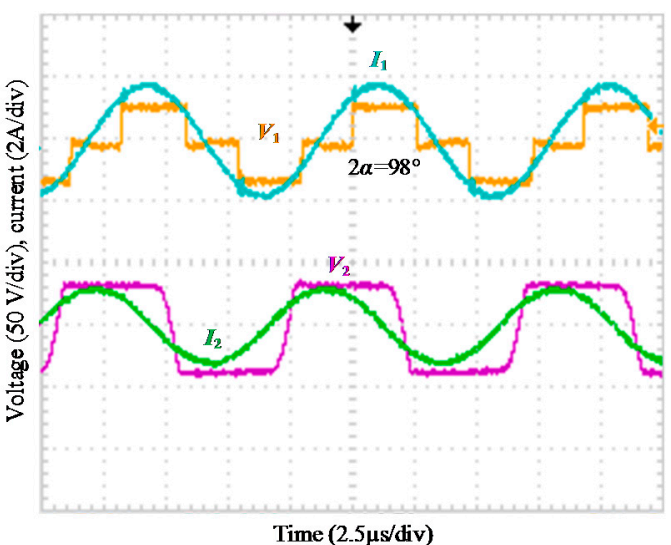

(a)

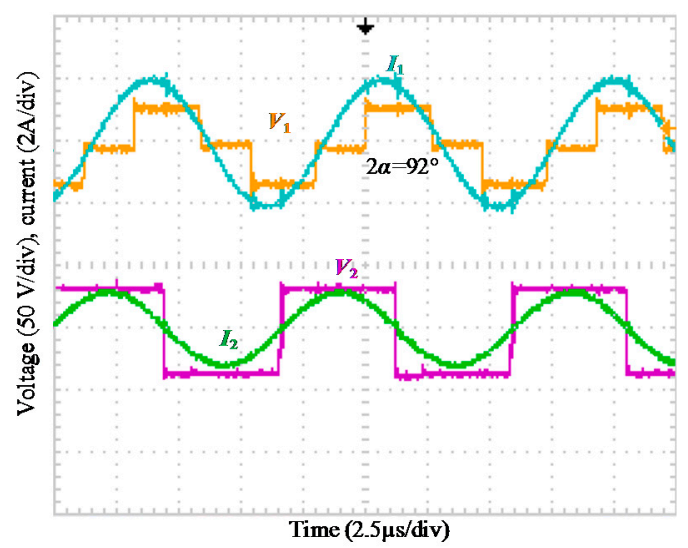

(b)

Figure 15. Cont. 


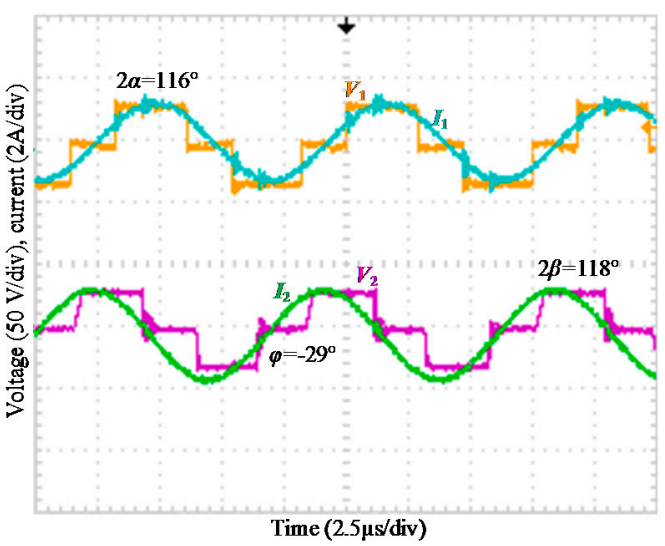

(c)

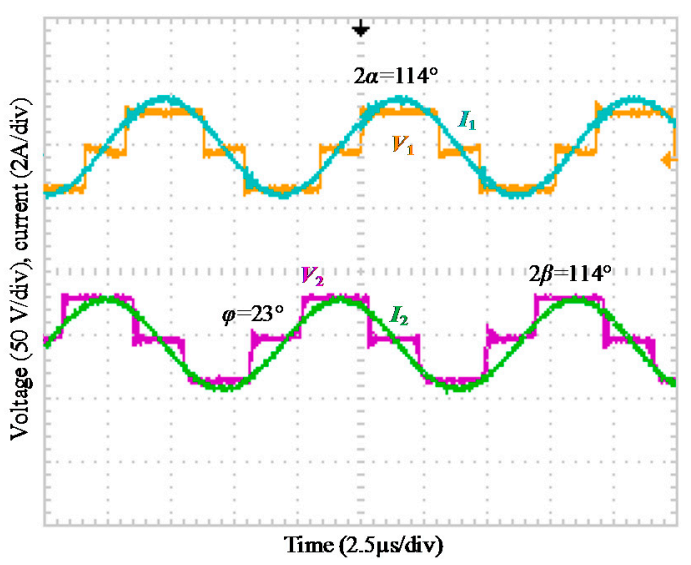

(d)

Figure 15. Experimental waveforms: (a) uncontrolled rectifier; (b) synchronous rectifier; (c) additional inductive reactance compensation and load transformation by MDPC; (d) additional capacitive reactance compensation and load transformation by MDPC.

The experimental results of $\alpha, \beta$ and $\varphi$ vs. $X_{2}$ are shown in Figure 16. $\alpha$ is higher than the simulation value due to the lower power transfer efficiency. The trends of $\beta$ and $\varphi$ agree with the simulation results.

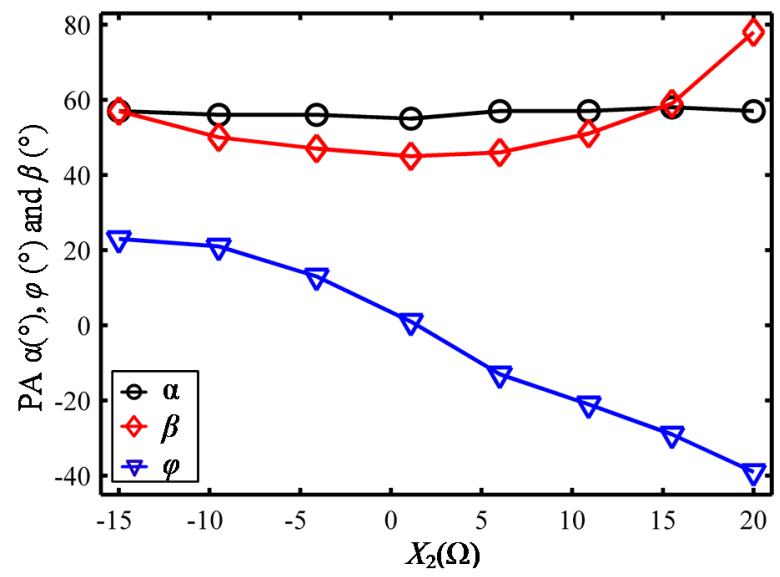

Figure 16. Experimental results of PAs versus additional reactance under MDPC.

The DC-DC experimental efficiencies are summarized in Figure 17. Figure 17a illustrates the experimental DC-DC efficiencies versus the load when $C_{2}=5.6 \mathrm{nF}$. When $R_{L}$ approaches the optimal value $24.8 \Omega, \beta$ is set at $90^{\circ}$. The synchronous rectifier system and the proposed method obtain the same overall efficiency of $80.4 \%$ with $21 \mathrm{~cm}$ distance between the transmitting and receiving coils. As $R_{L}$ deviates from the optimal value, the experimental efficiencies of the uncontrolled and synchronous rectifier systems decrease significantly, whereas the efficiency of the proposed system almost remains unchanged. Experimental results of additional reactance compensation are shown in Figure $17 \mathrm{~b}$, where $R_{L}$ is $50 \Omega$. The additional reactance requires higher apparent power and brings about efficiency degradation. Secondary PAs compensate the reactance and transforms the load simultaneously, thus the proposed system can attain higher efficiency than the other systems. 


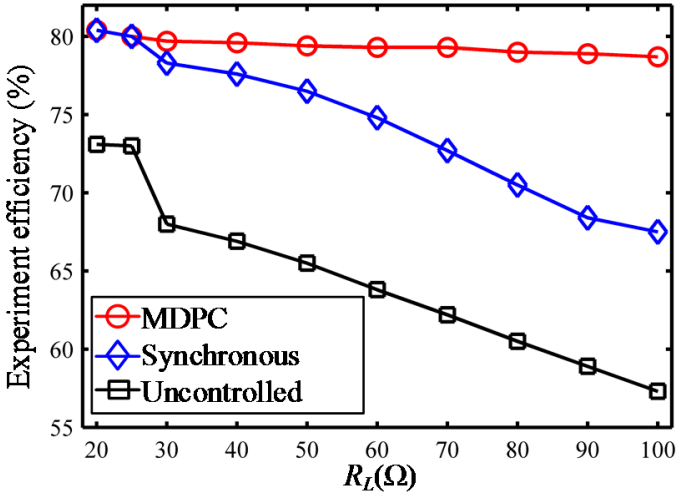

(a)

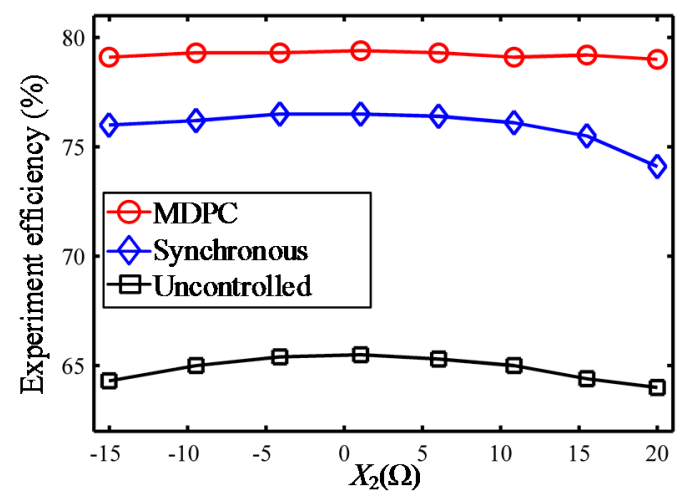

(b)

Figure 17. Experimental efficiency comparison of different methods: (a) experimental efficiency versus load; (b) experimental efficiency versus additional reactance.

Switches, filtering capacitors, connecting wires and the surrounding components consume some energy. These additional losses take up a large proportion when the transferred power is low. Thus, an efficiency gap between the simulation results and experimental results are observed. Similar comparable gaps can be found in previous works. In [17], the power transfer efficiency is less than $65 \%$ when the transferred power is $100 \mathrm{~W}$, whereas the overall efficiency is $92 \%$ with a $450-\mathrm{W}$ load. An increase in the transferred power level can improve the overall system efficiency and gaps between the experimental result and simulations are reduced.

\subsection{Discussion}

DC-DC efficiencies from the simulations and experiments with the same system specifications are summarized in Table 2. The proposed MDPC system achieves the highest simulated efficiency of $94.1 \%$ and the highest experimental efficiency of $80.4 \%$. The largest efficiency drops observed during large load and additional reactance variations are $0.6 \%$ for the simulation system and $1.7 \%$ for the experimental setup, respectively. In contrast, the other methods suffer from maximal $12.7 \%$ and $16.4 \%$ simulated and experimental efficiency drops, respectively. The proposed MDPC method can make full utilization of the three PAs for output regulation, reactance compensation and load transformation. It shows superiority to the other methods in terms of efficiency.

Table 2. DC-DC efficiencies of simulations and experiments.

\begin{tabular}{cccc}
\hline Header & Uncontrolled Rectifier & Synchronous Rectifier & MDPC \\
\hline Maximum simulated efficiency (\%) & 83.3 & 94.1 & 94.1 \\
Minimum simulated efficiency (\%) & 71.6 & 87.5 & 93.5 \\
Simulated efficiency drops (\%) & 12.7 & 6.6 & 0.6 \\
Maximum experimental efficiency (\%) & 73.1 & 80.4 & 80.4 \\
Minimum experimental efficiency (\%) & 57.3 & 64 & 78.7 \\
Experimental efficiency drops (\%) & 15.8 & 16.4 & 1.7 \\
\hline
\end{tabular}

Secondary PC regulates equivalent resistance to obtain the optimal value and the extracted power remains almost unchanged. Therefore, primary PA requires few adjustments. This can mitigate the drawback of time delay in communication and benefits for dual-side regulation. In other words, only dual-side regulation can realize load transformation and output regulation simultaneously.

\section{Conclusions}

In this paper, the MDPC method in a SS WPT system is proposed, with which the voltage regulation, load transformation and additional reactance compensation are achieved simultaneously. 
The presented work addresses the problem of performance degradations caused by additional DC-DC converters, non-identical resonances and load mismatch, which increased the power transfer efficiency and insured a compact design. The additional reactance and mutual inductance estimation methods were realized based on DC information sampling. The phase detection method modulated phase information onto a DC voltage signal. In addition, a novel synchronization method consisting of a comparator and an isolator performed well at a frequency of $100 \mathrm{kHz}$. A simulated efficiency of $94.1 \%$ and an experimental efficiency of $80.4 \%$ were attained while delivering $18 \mathrm{~W}$ power with $21 \mathrm{~cm}$ distance between the transmitting and receiving coils.

Supplementary Materials: The following are available online at www.mdpi.com/1996-1073/10/10/1588/s1.

Acknowledgments: The authors acknowledge support from the National Natural Science Foundation of China grant 51277120 and United Foundation of NSFC-Henan grant U1604136.

Author Contributions: Xin Liu proposed the main idea, designed and performed the experiments, and wrote the manuscript. Tianfeng Wang, Xijun Yang and Nan Jin double-checked and discussed the results and the whole manuscript. Houjun Tang and Xijun Yang contributed to the discussion of this study. All authors have approved the final version of this manuscript.

Conflicts of Interest: The authors declare no conflict of interest.

\section{References}

1. Kurs, A.; Karalis, A.; Moffatt, R.; Joannopoulos, J.D.; Fisher, P.; Soljacic, M. Wireless power transfer via strongly coupled magnetic resonances. Science 2007, 317, 83-86. [CrossRef] [PubMed]

2. Sample, A.P.; Meyer, D.A.; Smith, J.R. Analysis, experimental results, and range adaptation of magnetically coupled resonators for wireless power transfer. IEEE Trans. Ind. Electron. 2011, 58, 544-554. [CrossRef]

3. Lu, Y.; Ma, D.B. Wireless Power Transfer System Architectures for Portable or Implantable Applications. Energies 2016, 9, 1087. [CrossRef]

4. Wang, Z.; Wei, X.; Dai, H. Design and Control of a 3 kW Wireless Power Transfer System for Electric Vehicles. Energies 2016, 9, 10. [CrossRef]

5. Lee, S.-H.; Kim, J.-H.; Lee, J.-H. Development of a 60 kHz, 180 kW, Over 85\% Efficiency Inductive Power Transfer System for a Tram. Energies 2016, 9, 1075. [CrossRef]

6. Bashirullah, R. Wireless implants. IEEE Microw. Mag. 2010, 11, S14-S23. [CrossRef]

7. Wei, X.; Wang, Z.; Dai, H. A Critical Review of Wireless Power Transfer via Strongly Coupled Magnetic Resonances. Energies 2014, 7, 4316-4341. [CrossRef]

8. Kim, J.; Kim, J.; Kong, S.; Kim, H.; Suh, I.-S.; Suh, N.P.; Cho, D.-H.; Kim, J.; Ahn, S. Coil design and shielding methods for a magnetic resonant wireless power transfer system. Proc. IEEE 2013, 101, 1332-1342. [CrossRef]

9. Zhong, W.X.; Hui, S.Y.R. Maximum energy efficiency tracking for wireless power transfer systems. IEEE Trans. Power Electron. 2015, 30, 4025-4034. [CrossRef]

10. Hu, P.; Ren, J.; Li, W. Frequency-Splitting-Free Synchronous Tuning of Close-Coupling Self-Oscillating Wireless Power Transfer. Energies 2016, 9, 491. [CrossRef]

11. Mai, R.; Ma, L.; Liu, Y.; Yue, P.; Cao, G.; He, Z. A Maximum Efficiency Point Tracking Control Scheme Based on Different Cross Coupling of Dual-Receiver Inductive Power Transfer System. Energies 2017, $10,217$. [CrossRef]

12. Fu, M.; Yin, H.; Zhu, X.; Ma, C. Analysis and tracking of optimal load in wireless power transfer systems. IEEE Trans. Power Electron. 2015, 30, 3952-3963. [CrossRef]

13. Ahn, D.; Hong, S. Wireless power transfer resonance coupling amplification by load-modulation switching controller. IEEE Trans. Ind. Electron. 2015, 62, 898-909. [CrossRef]

14. Berger, A.; Agostinelli, M.; Vesti, S.; Oliver, J.A.; Cobos, J.A.; Huemer, M. A Wireless Charging System Applying Phase-Shift and Amplitude Control to Maximize Efficiency and Extractable Power. IEEE Trans. Power Electron. 2015, 30, 6338-6348. [CrossRef]

15. Li, H.; Li, J.; Wang, K.; Chen, W.; Yang, X. A maximum efficiency point tracking control scheme for wireless power transfer systems using magnetic resonant coupling. IEEE Trans. Power Electron. 2015, 30, 3998-4008. [CrossRef] 
16. Diekhans, T.; De Doncker, R.W. A dual-side controlled inductive power transfer system optimized for large coupling factor variations and partial load. IEEE Trans. Power Electron. 2015, 30, 6320-6328. [CrossRef]

17. Bac, X.N.; Vilathgamuwa, D.M.; Foo, G.H.B.; Wang, P.; Ong, A.; Madawala, U.K.; Trong, D.N. An EfficiencyOptimization Scheme for Bidirectional Inductive Power Transfer Systems. IEEE Trans. Power Electron. 2015, 30, 6310-6319.

18. Thrimawithana, D.J.; Madawala, U.K.; Neath, M. A Synchronization Technique for Bidirectional IPT Systems. IEEE Trans. Ind. Electron. 2013, 60, 301-309. [CrossRef]

19. Madawala, U.K.; Thrimawithana, D.J. A Bidirectional Inductive Power Interface for Electric Vehicles in V2G Systems. IEEE Trans. Ind. Electron. 2011, 58, 4789-4796. [CrossRef]

20. Colak, K.; Asa, E.; Bojarski, M.; Czarkowski, D.; Onar, O.C. A Novel Phase-Shift Control of Semibridgeless Active Rectifier for Wireless Power Transfer. IEEE Trans. Power Electron. 2015, 30, 6288-6297. [CrossRef]

21. Mai, R.; Liu, Y.; Li, Y.; Yue, P.; Cao, G.; He, Z. An Active Rectifier Based Maximum Efficiency Tracking Method Using an Additional Measurement Coil for Wireless Power Transfer. IEEE Trans. Power Electron. 2017, 99, 1. [CrossRef]

22. Kobayashi, D.; Imura, T.; Hori, Y. Real-time coupling coefficient estimation and maximum efficiency control on dynamic wireless power transfer for electric vehicles. In Proceedings of the IEEE PELS Workshop Emerging Technologies: Wireless Power, Daejeon, Korea, 5-6 June 2015.

23. Yin, J.; Lin, D.; Parisini, T.; Hui, S.Y. Front-End Monitoring of the Mutual Inductance and Load Resistance in a Series-Series Compensated Wireless Power Transfer System. IEEE Trans. Power. Electron. 2016, 31, 7339-7352. [CrossRef]

24. Li, Y.; Mai, R.; Lin, T.; Sun, H.; He, Z. A Novel WPT System Based on Dual Transmitters and Dual Receivers for High Power Applications: Analysis, Design and Implementation. Energies 2017, 10, 174. [CrossRef]

25. Thrimawithana, D.J.; Madawala, U.K.; Yu, S. Design of a bi-directional inverter for a wireless V2G system. In Proceedings of the IEEE International Conference on Sustainable Energy Technologies (ICSET), Kandy, Sri Lanka, 6-9 December 2010; pp. 1-5.

(C) 2017 by the authors. Licensee MDPI, Basel, Switzerland. This article is an open access article distributed under the terms and conditions of the Creative Commons Attribution (CC BY) license (http:/ / creativecommons.org/licenses/by/4.0/). 